\title{
Genetically Diverse Filoviruses in Rousettus and Eonycteris spp. Bats, China, 2009 and 2015
}

\author{
Xing-Lou Yang, ${ }^{1}$ Yun-Zhi Zhang, ${ }^{1}$ Ren-Di Jiang, ${ }^{1}$ \\ Hua Guo, Wei Zhang, Bei Li, Ning Wang, Li Wang, \\ Cecilia Waruhiu, Ji-Hua Zhou, Shi-Yue Li, \\ Peter Daszak, Lin-Fa Wang, Zheng-Li Shi
}

Genetically divergent filoviruses detected in Rousettus and Eonycteris spp. bats in China exhibited $61 \%-99 \%$ nt identity with reported filoviruses, based on partial replicase sequences, and they demonstrated lung tropism. Co-infection with 4 different filoviruses was found in 1 bat. These results demonstrate that fruit bats are key reservoirs of filoviruses.

$\mathrm{F}$ loviruses (family Filoviridae) are nonsegmented, negative-strand RNA viruses belonging to 3 genera: Marburgvirus, Ebolavirus, and Cuevavirus. Marburgvirus comprises 1 species, Marburg marburgvirus, which includes Marburg virus (MARV) and Ravn virus. Ebolavirus comprises 5 species, Zaire ebolavirus (ZEBOV), Sudan ebolavirus, Bundibugyo ebolavirus, Taï Forest ebolavirus, and Reston virus (RESTV). Cuevavirus comprises 1 species, Lloviu cuevavirus (1). Filovirus-associated diseases, especially those caused by ZEBOV and MARV, are recognized as a major threat to public health, causing high rates of death among humans and nonhuman primates.

Bats have been implicated as natural reservoirs for filoviruses $(2,3)$ on the basis of serologic evidence from 19 bat species in 8 countries across Asia, Africa, and Europe (2,4-9). In addition, filovirus RNA has been detected in 8 bat species from 7 countries in the same regions $(2-4,10-13)$. Outbreaks of Marburg hemorrhagic fever among miners in Uganda in 2007 were traced to bat MARV (11). In addition, we previously discovered filovirus antibodies in several bat species in China (14). This finding was further confirmed by $\mathrm{He}$ et al., who detected filovirus RNA in brown fruit bats (Rousettus leschenaultii) in China (10). Considering the diversity of bat species in

Author affiliations: Chinese Academy of Sciences, Wuhan, China (X.-L. Yang, R.-D. Jiang, H. Guo, W. Zhang, B. Li, N. Wang, L. Wang, C. Waruhiu, Z.-L. Shi); Dali University, Dali, China (Y.-Z. Zhang); University of Chinese Academy of Sciences, Beijing, China (R.-D Jiang, H. Guo, N. Wang); Yunnan Institute of Endemic Diseases Control and Prevention, Dali (Y.-Z. Zhang, J.-H. Zhou); Wuhan University, Wuhan (S.-Y. Li); EcoHealth Alliance, New York, New York, USA (P. Daszak); Duke-NUS Graduate Medical School, Singapore (L.-F. Wang)

DOI: http://dx.doi.org/10.3201/eid2303.161119 the world, long-term surveillance of bat filoviruses is essential for better understanding of distribution, diversity, and ecology of these viruses. We conducted a study to determine the diversity of filoviruses among bats in Yunnan Province, China.

\section{The Study}

We captured 150 apparently healthy adult bats from 2 caves in Yunnan Province, China: 1 in Jinghong City in November 2009, and 1 in Mengla County in December 2015 (Table 1; Figure 1). The bat species we collected were Hipposideros armiger, Aselliscus stoliczkanus, Myotis ricketti, Rhinolophus Monoceros, Miniopterus fuscus, Ia io, Eonycteris spelaea, and Rousettus sp. We humanely killed all bats and collected their hearts, intestines, lungs, spleens, kidneys, livers, brains, and blood for testing. We used 2 methods to analyze bat lung tissues for presence of filovirus RNA: first, we used nested PCR with the primers FV F1/R1 and FV F2/R2 (10), and next, we used quantitative PCR (qPCR) with 3 groups of qPCR with primers and probes designed from viral sequences obtained in this study (online Technical Appendix Table, https://wwwnc. cdc.gov/EID/article/23/3/16-1119-Techapp1.pdf).

Using degenerate nested PCR, we detected filovirus RNA in 15 fruit bat specimens (E. spelaea and Rousettus sp.); the specimens comprised $10(23.3 \%)$ of 43 E. spelaea and Rousettus sp. collected in 2009 and $5(11.9 \%)$ of 42 collected in 2015. Using qPCR, we detected filovirus RNA in 20 specimens from E. spelaea $(\mathrm{n}=4)$ and Rousettus $\mathrm{sp}$. $(\mathrm{n}=16)$ bats: $10(23.3 \%)$ of the bats were collected in 2009 and $10(23.8 \%)$ in 2015 . No filovirus was detected in other bat species studied (Table 1). The 310-bp L gene sequences (GenBank accession nos. KX371873-KX371890) exhibited 65\%-99\% nt identity among themselves and 61\%-99\% nt identity with known filoviruses. Phylogenetic analysis showed that the sequences from the bats formed 3 independent groups, groups 1-3. Groups 1 and 2 comprised 6 and 11 sequences, respectively, all of which were obtained in this study (Figure 2). Group 3 comprised 2 sequences, 1 from this study and 1 previously published (10). Pairwise distance analysis indicated that sequences in group 1 share the highest nucleotide identity $(75 \%-78 \%)$ with MARV and those in group 2 share the highest identity $(69 \%-74 \%)$ with Ravn virus. The 2 sequences in group 3 are highly similar and share $66 \%-70 \%$ nt identity with other filovirus species. Of note, 1 bat specimen (no. 9447) was co-infected

${ }^{1}$ These authors contributed equally to this article. 
Table 1. Filovirus infection detected in bat samples by PCR, ELISA, and Western blot, Yunnan Province, China, 2009 and $2015^{\star}$

\begin{tabular}{|c|c|c|c|c|c|c|}
\hline \multirow{3}{*}{$\begin{array}{l}\text { Bat species, by year and month of } \\
\text { collection/location }\end{array}$} & \multicolumn{6}{|c|}{ No. positive/no. tested (\%) } \\
\hline & \multirow[b]{2}{*}{ RT-PCR } & \multirow{2}{*}{$\begin{array}{l}\text { Quantitative } \\
\text { PCR }\end{array}$} & \multicolumn{2}{|c|}{ ELISA $†$} & \multicolumn{2}{|c|}{ Western blot $\dagger$} \\
\hline & & & ZEBOV & RESTV & ZEBOV & RESTV \\
\hline \multicolumn{7}{|l|}{2009 Nov/Jinghong City } \\
\hline Hipposideros armiger & $0 / 15$ & $0 / 15$ & $0 / 15$ & $0 / 15$ & $0 / 15$ & $0 / 15$ \\
\hline Rhinolophus monoceros & $0 / 4$ & $0 / 4$ & $0 / 4$ & $0 / 4$ & $0 / 4$ & $0 / 4$ \\
\hline la io & $0 / 3$ & $0 / 3$ & $0 / 3$ & $0 / 3$ & $0 / 3$ & $0 / 3$ \\
\hline Miniopterus fuscus & $0 / 1$ & $0 / 1$ & $0 / 1$ & $0 / 1$ & $0 / 1$ & $0 / 1$ \\
\hline Myotis ricketti & $0 / 27$ & $0 / 27$ & $1 / 27(3.7)$ & $1 / 27(3.7)$ & $1 / 27(3.7)$ & $1 / 27(3.7)$ \\
\hline Eonycteris spelaea and Rousettus sp. & $10 / 43(23.3)$ & $10 / 43(23.3)$ & $5 / 43(11.6)$ & $6 / 43(13.9)$ & $2 / 43(4.6)$ & $2 / 43(4.6)$ \\
\hline \multicolumn{7}{|l|}{2015 Dec/Mengla County } \\
\hline Aselliscus stoliczkanus & $0 / 15$ & $0 / 15$ & $0 / 15$ & $0 / 15$ & $0 / 15$ & $0 / 15$ \\
\hline E. spelaea and Rousettus sp. & $5 / 42(11.9)$ & $10 / 42(23.8)$ & $14 / 25(56)$ & $7 / 25(28)$ & $11 / 25(44)$ & $4 / 25(16)$ \\
\hline
\end{tabular}

*EBOV, Zaire ebolavirus; RESTV, Reston virus.

†ELISA and Western blot results for samples collected in 2009 were from a previous study (14).

with 4 different filovirus strains (BtFiloYN9447-1 to 9447-4) with high divergence (Figure 2; online Technical Appendix Figure 1). To further determine the phylogenetic relationship of these viruses with known filoviruses, we amplified more $\mathrm{L}$ gene sequence $(1,475 \mathrm{bp})$ for strains BtFiloYN2162 and BtFiloYN9447-1. Similar to the 310bp sequences, the 1,475-bp sequence of the BtfiloYN2162 shared $99 \%$ identity with BtDH04 at the nucleotide level, the 1,475-bp sequence of BtFiloYN9447-1 shared 62\%$71 \%$ with known filoviruses.

To determine the tissue tropism of these viruses, we performed $\mathrm{qPCR}$ with primers and probes designed for each of the 3 different groups (online Technical Appendix Table). Results showed that filoviruses were mainly located in the lung and that genome copy numbers ranged from 29 to $523,582 / \mathrm{mg}$ of tissue (Table 2). Only 2 bat blood samples (nos. 2202 and 9447) were positive for filovirus RNA; 5 samples (nos. 2202, 2188, 9434, 9442, and 9447) contained filoviruses with more widespread tissue tropism. We were unable to isolate virus from PCR-positive samples by using Vero-E6 cells.

To detect filovirus IgG and IgM, we expressed Histagged truncated nucleoproteins from RESTV or ZEBOV in Escherichia coli and used them as antigens (online

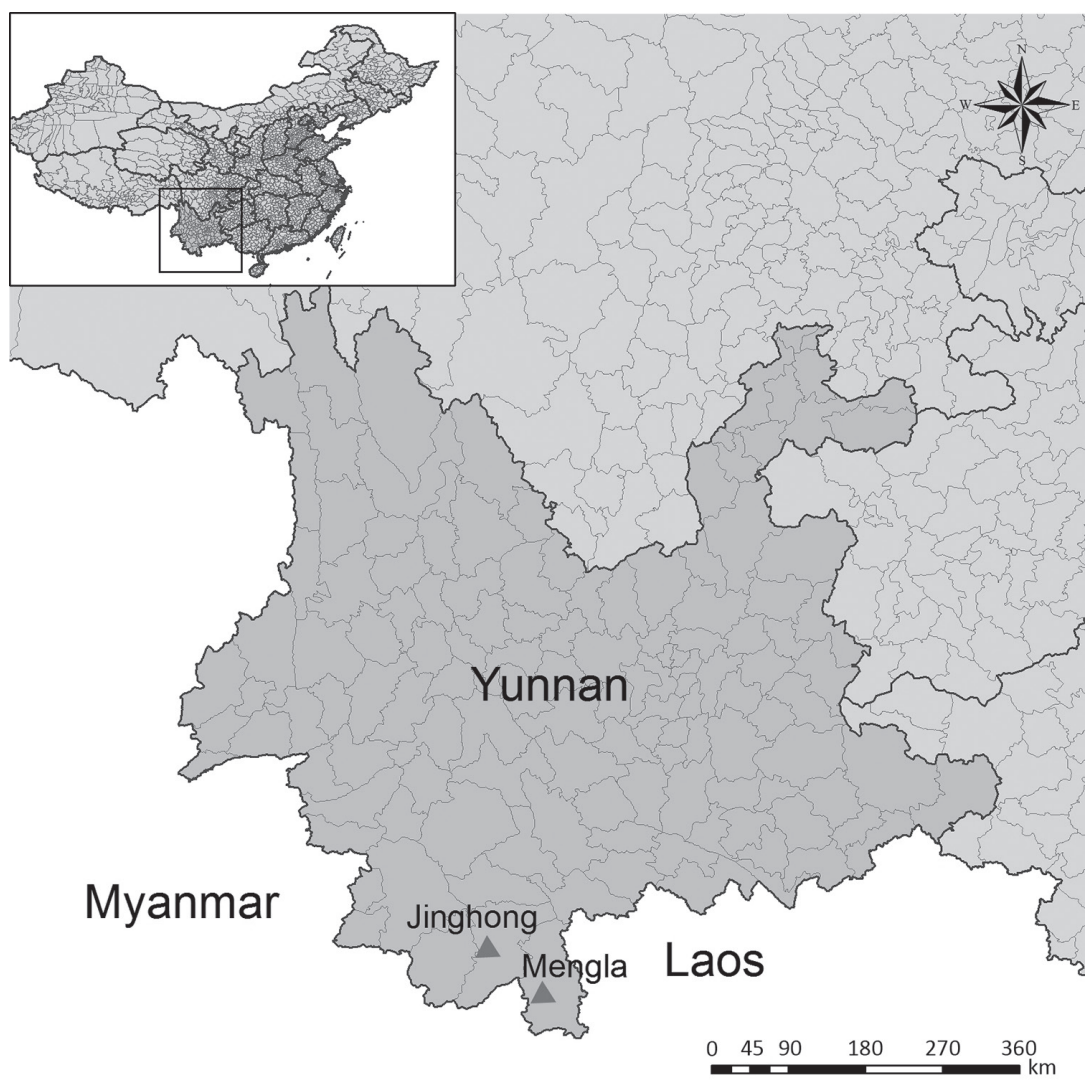

Figure 1. Bat collection sites for a study on genetically diverse filoviruses in Rousettus and Eonycteris spp. bats in China. Triangles indicate Jinghong City and Mengla County, Yunnan Province, where 150 apparently healthy adult bats were collected from 2 caves in November 2009 (Jinghong City) and December 2015 (Mengla County). Inset map shows the location of Yunnan Province in China. 
Table 2. Virus tropism and quantification in different tissues of Eonycteris spelaea and Rousettus sp. bats, China, 2009 and 2015

\begin{tabular}{|c|c|c|c|c|}
\hline Sample no. & Species & Sex & Primer group* & $\begin{array}{l}\text { Positive organs (viral genome copies } / \mathrm{mg} \\
\text { tissue or viral genome copies } / \mu \mathrm{L} \text { blood) }\end{array}$ \\
\hline 2162 & E. spelaea & $\mathrm{M}$ & 3 & Lung (119) \\
\hline 2176 & Rousettus sp. & $\mathrm{M}$ & 2 & Lung $(2,103)$ \\
\hline 2180 & Rousettus sp. & $\mathrm{F}$ & 2 & Lung (42) \\
\hline 2181 & Rousettus sp. & M & 2 & Lung (202) \\
\hline 2187 & Rousettus sp. & $\mathrm{F}$ & 1 & Lung (46) \\
\hline 2188 & Rousettus sp. & $\mathrm{F}$ & 1 & Lung (43), liver (400), kidney (42) \\
\hline 2190 & Rousettus sp. & M & 2 & Lung (195) \\
\hline 2196 & Rousettus sp. & $\mathrm{F}$ & 1 & Lung (123) \\
\hline 2199 & Rousettus sp. & $\mathrm{F}$ & 1 & Lung (74) \\
\hline 2202 & Rousettus sp. & $\mathrm{F}$ & 1 & $\begin{array}{l}\text { Lung (864), liver (368), kidney (342), intestine } \\
\text { (254), heart (807), blood (1) }\end{array}$ \\
\hline 9428 & Rousettus sp. & $\mathrm{F}$ & 2 & Lung (156) \\
\hline 9434 & Rousettus sp. & $\mathrm{F}$ & 1 & $\begin{array}{l}\text { Lung }(554) \text {, spleen }(3,014) \text {, kidney }(380) \text {, } \\
\text { heart }(320) \text {, intestine }(2751)\end{array}$ \\
\hline 9435 & Rousettus sp. & $\mathrm{F}$ & 2 & Lung (127) \\
\hline 9442 & E. spelaea & M & 2 & Lung (180), spleen (88) \\
\hline 9445 & Rousettus sp. & M & 1 & Lung (143) \\
\hline 9447 & Rousettus sp. & $\mathrm{F}$ & 2 & Lung (132), liver (154), spleen (661) \\
\hline 9447 & Rousettus sp. & $\mathrm{F}$ & 1 & $\begin{array}{c}\text { Lung }(106,606) \text {, liver }(220,051) \text {, spleen } \\
(523,582), \text { kidney }(41,653), \text { brain }(4,885) \text {, } \\
\text { heart }(17,982) \text {, intestine }(11,788) \text {, blood }(485)\end{array}$ \\
\hline 9454 & Rousettus sp. & M & 1 & Lung (448) \\
\hline 9457 & E. spelaea & M & 1 & Lung (52) \\
\hline 9459 & Rousettus sp. & $\mathrm{F}$ & 2 & Lung (182) \\
\hline 9463 & E. spelaea & $M$ & 2 & Lung (114) \\
\hline
\end{tabular}

Technical Appendix). In this experiment, we used 25 bat samples from 2015 that had enough serum volume for testing; 14 samples showed a strong cross-reaction with the ZEBOV nucleoprotein, and among them, 7 showed a weak cross-reaction with RESTV nucleoprotein. We used Western blotting to confirm these results; 11 of the 25 samples were positive for ZEBOV nucleoprotein and 4 for RESTV nucleoprotein (Table 1; online Technical Appendix Figure 2). No samples overlapped between those identified as positive by PCR and those identified as positive by serologic testing. Results of a serum neutralization assay with HIV pseudovirus carrying the ZEBOV glycoprotein showed that the ELISA-positive samples had no cross-neutralization activity to the pseudovirus (14).

\section{Conclusions}

We detected novel filovirus sequences with high divergence in E. spelaea and Rousettus sp. bats in China. Phylogenetic analysis of partial sequences suggested that at least 3 distinct groups of filovirus are circulating in fruit bats in China. The distances between these sequences indicates that the 3 groups may represent 3 novel species or genera. Of interest, we detected antibodies reacting more strongly to ZEBOV than RESTV nucleoprotein in some filovirus RNA-negative samples, suggesting that the bats were infected with another/other filovirus(es) cross-reactive with ZEBOV nucleoprotein or that nucleoproteins of the novel filoviruses were cross-reactive with ZEBOV and RESTV nucleoproteins.
The bat samples in this study were collected from 2 caves in 2009 and 2015, respectively; the caves are $\approx 200 \mathrm{~km}$ metric apart. Across the 2 different years and locations, we detected closely related viruses and found 1 bat that was acutely co-infected by 4 different filoviruses; this finding suggests that these viruses have been circulating in the 2 bat species and that densely populated bat caves provide opportunity for crossinfection with different viruses. However, considering the migration ability of the fruit bat, we cannot exclude the possibility that there are exchanges of virus between the bats in these two caves. Longitudinal surveillance with tracking tags may help to better understand the spatial-temporal distribution of these viruses in bat populations.

In previous reports, filoviruses were primarily detected in liver and spleen tissues $(4,15)$. In our study, we primarily detected filoviruses in the lung. We suspect that lung tissues are the major target for these bat filoviruses. Thus, these filoviruses may have the potential to be transmitted through the respiratory tract.

These results will be helpful in providing a better understanding of the distribution and diversity of filoviruses, which may have implications for public health. Considering their feeding habitats, fruit bats are often in close contact with domestic animals and human populations. It is therefore necessary to establish longterm and proactive surveillance of these viruses and related diseases. 


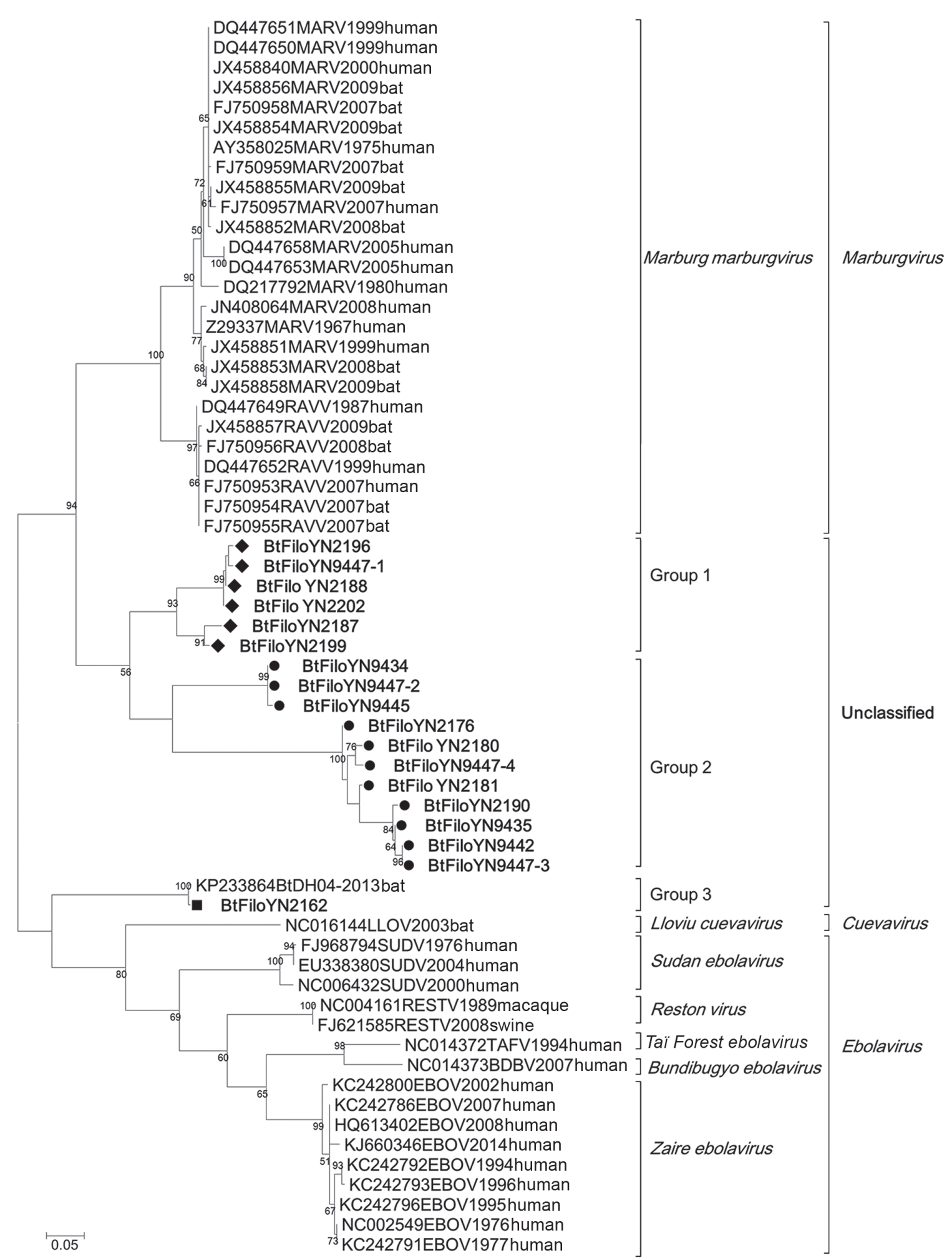

Figure 2. Phylogenetic analysis of filovirus isolates collected in study of genetically diverse filoviruses in Rousettus and Eonycteris spp. bats in China, compared with reference isolates. Analysis was based on a 310-bp segment of the filovirus $L$ gene. Bootstrap values lower than 50 are not shown. The maximum-likelihood tree was constructed based on the 310-bp segment with 1,000 bootstrap replicates. The sequences obtained in this study are marked with a triangle (group 1), black dot (group 2), or rectangle [group 3). Sequences from GenBank are listed by their accession numbers, followed by the virus name, collection year, and host. Scale bar indicates nucleotide substitutions per site. 
This work was funded by a joint National Natural Science Foundation of China grant (81290341) and the China Mega-Project for Infectious Disease grant (2014ZX10004001-003) from the Minister of Science and Technology of the People's Republic of China (to Z-L.S.); a Scientific and Technological Basis Special Project grant (2013FY113500; to Z-L.S. and Y-Z.Z.); a National Natural Science Foundation of China grant (81260437) and State Key Laboratory for Infectious Diseases Prevention and Control grant (2013SKLID302) (to Y-Z.Z.); and a United States Agency for International Development Emerging Pandemic Threats PREDICT project grant (cooperative agreement AID-OAA-A-14-00102; to P.D.D.).

Dr. Yang is as a research assistant at Wuhan Institute of Virology, Chinese Academy of Sciences. His primary research interests include viral epidemiology and viral characterization of small mammals.

\section{References}

1. King AMQ, Adams MJ, Carstens EB, Lefkowitz EJ, editors. Virus taxonomy: ninth report of the International Committee on Taxonomy of Viruses. San Diego (CA): Academic Press; 2012.

2. Olival KJ, Hayman DT. Filoviruses in bats: current knowledge and future directions. Viruses. 2014;6:1759-88. http://dx.doi.org/ $10.3390 / \mathrm{v} 6041759$

3. Jayme SI, Field HE, de Jong C, Olival KJ, Marsh G, Tagtag AM, et al. Molecular evidence of Ebola Reston virus infection in Philippine bats. Virol J. 2015;12:107. http://dx.doi.org/10.1186/ s12985-015-0331-3

4. Leroy EM, Kumulungui B, Pourrut X, Rouquet P, Hassanin A, Yaba P, et al. Fruit bats as reservoirs of Ebola virus. Nature. 2005;438:575-6. http://dx.doi.org/10.1038/438575a

5. Swanepoel R, Smit SB, Rollin PE, Formenty P, Leman PA, Kemp A, et al.; International Scientific and Technical Committee for Marburg Hemorrhagic Fever Control in the Democratic Republic of Congo. Studies of reservoir hosts for Marburg virus. Emerg Infect Dis. 2007;13:1847-51. http://dx.doi.org/10.3201/ eid1312.071115
6. Kuzmin IV, Niezgoda M, Franka R, Agwanda B, Markotter W, Breiman RF, et al. Marburg virus in fruit bat, Kenya. Emerg Infect Dis. 2010;16:352-4. http://dx.doi.org/10.3201/ eid1602.091269

7. Taniguchi S, Watanabe S, Masangkay JS, Omatsu T, Ikegami T, Alviola P, et al. Reston Ebolavirus antibodies in bats, the Philippines. Emerg Infect Dis. 2011;17:1559-60.

8. Olival KJ, Islam A, Yu M, Anthony SJ, Epstein JH, Khan SA, et al Ebola virus antibodies in fruit bats, Bangladesh. Emerg Infect Dis. 2013;19:270-3. http://dx.doi.org/10.3201/eid1902.120524

9. Towner JS, Pourrut X, Albariño CG, Nkogue CN, Bird BH, Grard G, et al. Marburg virus infection detected in a common African bat. PLoS One. 2007;2:e764. http://dx.doi.org/10.1371/ journal.pone.0000764

10. He B, Feng Y, Zhang H, Xu L, Yang W, Zhang Y, et al. Filovirus RNA in Fruit Bats, China. Emerg Infect Dis. 2015;21:1675-7. http://dx.doi.org/10.3201/eid2109.150260

11. Towner JS, Amman BR, Sealy TK, Carroll SA, Comer JA, Kemp A, et al. Isolation of genetically diverse Marburg viruses from Egyptian fruit bats. PLoS Pathog. 2009;5:e1000536. http://dx.doi.org/10.1371/journal.ppat.1000536

12. Negredo A, Palacios G, Vázquez-Morón S, González F, Dopazo H, Molero F, et al. Discovery of an ebolavirus-like filovirus in Europe. PLoS Pathog. 2011;7:e1002304. http://dx.doi.org/10.1371/journal. ppat. 1002304

13. Amman BR, Carroll SA, Reed ZD, Sealy TK, Balinandi S, Swanepoel R, et al. Seasonal pulses of Marburg virus circulation in juvenile Rousettus aegyptiacus bats coincide with periods of increased risk of human infection. PLoS Pathog. 2012;8:e1002877. http://dx.doi.org/10.1371/journal. ppat. 1002877

14. Yuan J, Zhang Y, Li J, Zhang Y, Wang LF, Shi Z. Serological evidence of ebolavirus infection in bats, China. Virol J. 2012;9:236. http://dx.doi.org/10.1186/1743-422X-9-236

15. Brauburger K, Hume AJ, Mühlberger E, Olejnik J. Forty-five years of Marburg virus research. Viruses. 2012;4:1878-927. http://dx.doi.org/10.3390/v4101878

Address for correspondence: Zheng-Li Shi, Key Laboratory of Special Pathogens and Biosafety, Wuhan Institute of Virology, Chinese Academy of Sciences, Wuhan 430071, China; email: zlshi@wh.iov.cn

\section{EID Podcast:
Quiet Moment around the Campfire}

Frederic Remington was an American painter, sculptor, illustrator, and writer whose works frequently featured cowboys, Native Americans, soldiers, horses, bison, and other iconic features of the rapidly vanishing American West. The EID June, 2014 cover painting, commonly known as The Cigarette, was discovered in Remington's studio after his death. In this painting, four cowboys relax around a small outside a cabin. A plume of smoke rises toward the clear blue-green night sky flecked with a few stars, past a large skin hanging on the side of the cabin. The cabin does not overwhelm the painting but details such as the shadow under the roofline, the seams between logs, the softened edges of the structure, and the tautly stretched skin reveal Remington's deftness at rendering textures. His use of subdued colors punctuated by the reflected firelight underscores the quiet of the evening's respite following a long day's work. 